\title{
Endothelin-converting enzyme-1 promoter polymorphisms and susceptibility to sporadic late-onset Alzheimer's disease in a Chinese population
}

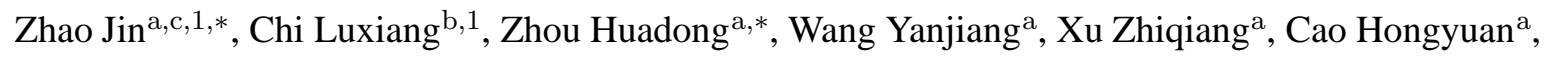 \\ Huang Lihua ${ }^{\mathrm{a}}$ and $\mathrm{Yi} \mathrm{Xu}^{\mathrm{a}}$ \\ ${ }^{a}$ Department of Neurology, Daping Hospital, Third Military Medical University, Chongqing 400042, China \\ ${ }^{\mathrm{b}}$ Department of Cardiology, Southwest Hospital, Third Military Medical University, Chongqing 400038, China \\ ${ }^{\mathrm{c}}$ Department of Haematology, Daping Hospital, Third Military Medical University, Chongqing 400042, China
}

\begin{abstract}
Endothelin converting enzyme (ECE-1) is a candidate Alzheimer disease susceptibility gene. It was previously reported that western individuals homozygous for the C-338A polymorphism (AA) within the ECE1 gene promoter region are at reduced risk of developing late onset Alzheimer disease (LOAD). A further polymorphism, T-839G, is present within the ECE1 promoter region but a potential association with LOAD has not been studied. We therefore studied possible associations between these ECE1 polymorphisms and LOAD in a Chinese population. Subjects comprised 376 Chinese LOAD patients and 376 age- and sex-matched controls; all subjects were typed for the ECE1 C-338A and the T-839G polymorphisms. We report that the frequency of the 338A allele was decreased in LOAD patients compared to controls (adjusted OR $=0.73 ; 95 \% \mathrm{CI}=0.54-0.98 ; P=0.03$ ). There was no significant association between T-839G genotype and LOAD, however the combined 839T/338A haplotype was significantly associated with decreased risk of $\operatorname{LOAD}(\mathrm{OR}=0.73 ; 95 \% \mathrm{CI}=0.57-0.93 ; P=0.01)$. This study argues that the ECE1 338A allele is protective against LOAD in a Chinese population.
\end{abstract}

Keywords: Alzheimer's disease, polymorphism, ECE-1

\section{Introduction}

Cerebral accumulation of $\beta$-amyloid peptide $(\mathrm{A} \beta)$ is thought to be central to the pathogenesis of Alzheimer's disease (AD) [1]. Endothelin-converting enzyme-1 (ECE- 1 ) is a candidate $\mathrm{A} \beta$-degrading enzyme in brain, and polymorphisms affecting expression levels of the ECE1 gene may therefore affect the risk of developing AD.

\footnotetext{
${ }^{1}$ These authors contributed equally to this study.

*Corresponding authors. Zhao Jin, Tel.: +86 13527525132; Fax: +86 23 68813806; E-mail: zhaojin78@126.com; Zhou Huadong, E-mail: zhouhuad@163.com.
}

The promoter region of the ECE1 gene contains a polymorphic site at position 338 (C-338A); alleles containing $\mathrm{A}$ at this position are associated with increased transcriptional activity in promoter-reporter assays in vitro as well as with increased ECE-1 mRNA expression in human neocortex [2]. It has also been reported in large case-control studies that individuals homozygous for C-338A (ie. AA) are at reduced risk of developing $\mathrm{AD}[3,4]$. A further functional polymorphism, T-839G, has also been identified within the ECE1 promoter region and gene alleles containing the 338A polymorphism (338A/839T and 338A/839G) were reported to have increased transcriptional activity [2].

A potential association between ECE1 T-839G polymorphisms and the risk of late onset AD (LOAD) has 
Table 1

Baseline characteristics of LOAD patients and controls

\begin{tabular}{|c|c|c|c|}
\hline \multirow[t]{2}{*}{ Characteristics } & LOAD & Controls & \multirow[t]{2}{*}{$P$} \\
\hline & $(n=376)$ & $(n=376)$ & \\
\hline $\operatorname{Age}^{\mathrm{a}}(\mathrm{y})$ & $68.2(62-94)$ & $68.4(62-94)$ & NS \\
\hline Sex (male), n (\%) & $123(32.7 \%)$ & $123(32.7 \%)$ & NS \\
\hline Education $(\leqslant 6 y)$ & $157(41.8 \%)$ & $107(28.5 \%)$ & $<0.001$ \\
\hline Smoking, n (\%) & $162(43.1 \%)$ & $90(23.9 \%)$ & $<0.001$ \\
\hline Drinking, n (\%) & $243(64.6 \%)$ & $164(43.6 \%)$ & $<0.001$ \\
\hline Hypertension, n (\%) & $211(56.1 \%)$ & $131(34.8 \%)$ & $<0.001$ \\
\hline Diabetes, n (\%) & $141(37.5 \%)$ & $68(18.1 \%)$ & $<0.001$ \\
\hline
\end{tabular}

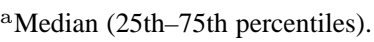

so far not been investigated. In present study we genotyped LOAD patients and control subjects in a Chinese population for their genotypes and haplotypes at the $\mathrm{C}$ 338A and T-839G polymorphic sites in order to study a possible association with LOAD.

\section{Material and methods}

\subsection{Patients and controls}

A cohort of 376 Chinese Han subjects with sporadic late-onset ( $>60$ years) Alzheimer's disease was recruited at the Division of Neurology of Daping Hospital (Chongqing, China). Dementia was diagnosed according to DSM-IV-R criteria [5]. Cognitive performance was assessed using the mini mental state evaluation and the global deterioration scale. All LOAD cases were defined as sporadic because reported family histories contained no first-degree relatives with dementia. Probable sporadic LOAD was diagnosed according to the NINCDS-ADRDA Work Group guidelines (revised) [6]. The control group comprised 376 unrelated and age- and sex-matched individuals randomly selected from the same area. These subjects had complete neurological, laboratory and medical examinations and were shown to be free of neurodegenerative disorders. The following risk factors for LOAD development were evaluated: low education, smoking, alcohol consumption, hypertension and diabetes. All patients and controls were free of anti-hypertensive treatment. Participants who had ever smoked within the 10 years prior to study entry were defined as smokers. All participants gave informed consent for the study and the outline of the study was approved by the Institutional Review Board.

\subsection{Genotyping of ECE1 polymorphisms}

After overnight fasting approximately $5 \mathrm{ml}$ samples of venous blood were drawn from each subject into tubes containing EDTA. Genomic DNA was extracted according to the salting-out procedure described by Miller et al. [7]. The ECE1 C-338A polymorphism was investigated according to the technique described by Funalot et al. and Scacchi et al. [4,8]. T-839G polymorphisms were detected by the same method. The PCR primers and restriction enzymes used in this work are summarized in Table 2.

\subsection{Statistical analysis}

Statistical analyses were conducted using the SPSS 13.0 program (SPSS Inc., Chicago, IL). HardyWeinberg equilibrium of the analyzed polymorphisms was tested in the study population by a $\chi 2$ goodness-offit test. Odds ratios (ORs) are reported with $95 \%$ confidence interval (CI) and P-values were calculated using the $\chi 2$-test. Logistic regression analysis was performed with respect to the C-338A or T-839G genotypes and the following covariates: low education, diabetes, hypertension, smoking and alcohol consumption. Linkage disequilibrium was tested using the LINKDOS program of Garnier-Gere and Dillmann [9]. Haplotype analysis was conducted using modules implemented in the Haplo.stats program [10].

\section{Results}

The clinical characteristics of all study participants are shown in Table 1. As expected, known risk factors for LOAD including low education, smoking, alcohol consumption, hypertension and diabetes were more common among cases than controls $(P<0.001)$.

The ECE1 C-338A and T-839G variants were in Hardy-Weinberg equilibrium $(P=0.293$ and $P=$ 0.697 respectively for C-338A LOAD patients and controls; $P=0.391$ and $P=0.582$ respectively for G839T LOAD patients and controls) and were in strong linkage disequilibrium (LD: $r^{2}=0.713$ ).

The distribution of C-338A genotypes (CC, CA and AA) was significantly different between LOAD patients $(81.9 \%, 16.8 \%$, and $1.3 \%)$ and controls $(59.8 \%$, $34.6 \%$, and $5.6 \% ; \chi 2=7.168, P=0.028)$, indicating a strong association between 338A alleles and LOAD (adjusted $\mathrm{OR}=0.73 ; 95 \% \mathrm{CI}=0.54-0.98 ; P=0.03$ ). 
Table 2

Primers sequences

\begin{tabular}{llll}
\hline Polymorphism & refSNP ID & Primer pairs Sense/Antisense & RE \\
\hline C-338A & rs213045 & 5/- TAG GGT TATAGG AGA GGG CTC AGG-3' & Tsp509I \\
& & 5/-AAG TAT CAG GAA GGT GCC CTC AAT-3' & \\
T-839G & rs213046 & 5/- CCCCTCACCCCTCTAAACCT -3' & AvaI \\
& & 5/- CACGGGATAAAGGAGCTGT-3/ & \\
\hline
\end{tabular}

There was no significant different in the distribution of ECE1 T-839G genotypes (CC, CA and AA) between LOAD patients $(51.6 \%, 38.8 \%$, and $9.6 \%)$ and controls (78.7\%, 19.7\%, and 1.6\%; $\chi 2=1.213, P=5.45)$. There was also no significant difference between genotype at position 839 and LOAD even after adjustment for known risk factors including education, diabetes, hypertension, smoking and alcohol consumption (adjusted $\mathrm{OR}=1.24 ; 95 \% \mathrm{CI}=0.87-1.79 ; P=0.28$ ) (Table 3).

The most frequent haplotype in the healthy Chinese control subjects was $839 \mathrm{~T} / 338 \mathrm{C}$, followed by $839 \mathrm{~T} / 338 \mathrm{~A}$ and $839 \mathrm{G} / 338 \mathrm{C}$. Similar haplotype frequencies were found in patients with LOAD. However, the combined 839T/338A haplotype was significantly associated with LOAD $(\mathrm{OR}=0.73 ; 95 \% \mathrm{CI}=0.57-$ 0.93; $P=0.01$ ) (Table 4).

\section{Discussion}

In the present case-control study we compared the distribution of C-338A and T-839G polymorphisms within the ECE1 gene between patients with LOAD and a control group of age- and sex-matched subjects without any neurodegenerative disorder. Both cohorts were extensively characterized with respect to known risk factors for LOAD in order to permit statistical adjustment. We report that ECE1 338A alleles and the $839 \mathrm{~T} / 338 \mathrm{~A}$ haplotype were significantly underrepresented in LOAD patients compared to control subjects.

Several genetic polymorphisms have been identified within the ECE1 gene promoter. Previous studies addressed potential associations with $\mathrm{AD}$, hypertension and coronary artery disease and further nonsynonymous ECE1 gene variants have also been studied [3, $4,8,11]$. Three different explanations have been put forward to explain the association between ECE1 gene polymorphisms and disease status. First, the polymorphisms may be biologically relevant to the disease; second, they are in linkage disequilibrium with biologically relevant variants elsewhere within the ECE1 gene; or third, these polymorphisms are in disequilibrium with genetic variants in other adjacent genes [12]. In vitro or in vivo expression studies of the mutant gene and protein will be required to address the functional relationship between the different polymorphisms and disease risk.

The 338A allele was previously reported to display increased transcriptional activity in transient transfection assays compared to promoter regions containing 338C. Variants containing 839G did not appear to affect transcriptional activity, although a synergistic effect on promoter efficacy was reported when $839 \mathrm{G}$ was combined with 338C [2]. In vivo, ECE1 mRNA expression levels were 2-fold higher in human prefrontal cortex of carriers of the 338A allele compared to non-carriers [3].

Expression of ECE-1 enzyme may be protective against $\mathrm{A} \beta$ deposition in Alzheimer disease. Overexpression of ECE-1 in Chinese hamster ovary cells lacking endogenous ECE activity was found to reduce extracellular $\mathrm{A} \beta$ concentrations by up to $90 \%$; this effect was completely abolished by treatment with the ECE-1 inhibitor phosphoramidon [13]. Moreover, recombinant soluble ECE-1 was shown to hydrolyze synthetic $\mathrm{A} \beta-(1-40)$ and $\mathrm{A} \beta-(1-42)$ [14]. Because both the $\mathrm{C}-338 \mathrm{~A}$ and $\mathrm{T}-839 \mathrm{G}$ polymorphisms affect expression levels of ECE-1 enzyme it would appear likely that different allelic variants at these sites might modulate the risk of $\mathrm{AD}$ development.

Although both 338A and $839 \mathrm{G}$ allele have been reported to increase ECE1 promoter activity, we found that the $839 \mathrm{G}$ allele was not significantly associated with protection against LOAD. This inconsistency was also found in relation to coronary artery disease. Wang et al. [15] reported that ECE1 338A alleles were associated with increased risk of coronary artery disease (adjusted OR $=1.58,95 \% \mathrm{CI}=1.07-2.32, P=0.020$ ) while, in contrast, Scacchia et al. [4] showed that 338A alleles were protective against coronary artery disease in subjects carrying the apolipoprotein $\mathrm{E}(A P O E) \mathrm{e}^{* 4}$ allele (adjusted OR $=0.51 ; 95 \% \mathrm{CI}=0.29-0.89 ; P=$ 0.018). Buhler et al. [11] found that subjects harboring the $839 \mathrm{G}$ allelic variant were at decreased risk of coronary artery disease (odds ratio $0.41 ; 95 \% \mathrm{CI}=0.18$ $0.90, P=0.024)$. No satisfactory explanation has yet been put forward to explain why increased ECE1 pro- 
Table 3

Distributions and the risk estimates for the variants of ECE1 C-338A and T-839G

\begin{tabular}{|c|c|c|c|c|c|c|c|c|}
\hline genotype & Controls $(\%)$ & $\operatorname{LOAD}(\%)$ & Crude OR & $95 \% \mathrm{CI}$ & $\mathrm{P}$ & Adjusted OR & $95 \% \mathrm{CI}$ & $\mathrm{P}$ \\
\hline \multicolumn{9}{|l|}{$\mathrm{T}-839 \mathrm{G}$} \\
\hline $\mathrm{TT}$ & $308(81.9 \%)$ & $296(78.7 \%)$ & 1 & & & & & \\
\hline TG & $63(16.8 \%)$ & $74(19.7 \%)$ & 1.22 & $(0.84-1.77)$ & 0.29 & 1.27 & $(0.86-1.83)$ & 0.31 \\
\hline GG & $5(1.3 \%)$ & $6(1.6 \%)$ & 1.25 & $(0.38-4.14)$ & 0.72 & 1.13 & $(0.25-3.98)$ & 0.73 \\
\hline $\begin{array}{l}\text { TG + GG } \\
\mathrm{C}-338 \mathrm{~A}\end{array}$ & $68(18.1 \%)$ & $80(21.3 \%)$ & 1.22 & $(0.85-1.76)$ & 0.27 & 1.24 & $(0.87-1.79)$ & 0.28 \\
\hline $\mathrm{CC}$ & $194(51.6 \%)$ & $225(59.8 \%)$ & 1 & & & & & \\
\hline $\mathrm{AC}$ & $146(38.8 \%)$ & $130(34.6 \%)$ & 0.77 & $(0.57-1.04)$ & 0.09 & 0.78 & $(0.58-1.07)$ & 0.09 \\
\hline AA & $36(9.6 \%)$ & $21(5.6 \%)$ & 0.50 & $(0.28-0.89)$ & 0.02 & 0.48 & $(0.25-0.87)$ & 0.02 \\
\hline $\mathrm{AA}+\mathrm{AC}$ & $182(48.4 \%)$ & $151(40.2 \%)$ & 0.72 & $(0.54-0.96)$ & 0.02 & 0.73 & $(0.54-0.98)$ & 0.03 \\
\hline
\end{tabular}

Patients and controls were matched by age and sex and data adjusted for low education, smoking, drinking, hypertension and diabetes.

Table 4

Distributions and the risk Estimates for ECE1haplotypes

\begin{tabular}{cccccc}
\hline haplotype $^{\mathrm{a}}$ & Controls(\%) & LOAD $(\%)$ & Crude OR & $95 \%$ CI & P \\
\hline $839 \mathrm{~T} / 338 \mathrm{C}$ & $469(62.4 \%)$ & $502(66.7 \%)$ & 1 & & \\
$839 \mathrm{~T} / 338 \mathrm{~A}$ & $210(27.9 \%)$ & $164(21.8 \%)$ & 0.73 & $0.57-0.93$ & 0.01 \\
$839 \mathrm{G} / 338 \mathrm{C}$ & $65(8.6 \%)$ & $78(10.4 \%)$ & 1.12 & $0.79-1.60$ & 0.53 \\
$839 \mathrm{G} / 338 \mathrm{~A}$ & $8(1.1 \%)$ & $8(1.1 \%)$ & 0.93 & $0.35-2.51$ & 0.89 \\
\hline
\end{tabular}

moter activity is associated with increased risk of coronary artery disease in some studies, and with decreased risk in others.

In the present study 338A alleles were associated with reduced risk of developing LOAD, whereas alleles containing $839 \mathrm{G}$ were not protective, even though both variants are thought to increase ECE1 promoter activity. These contrasting findings may possibly be explained by variations in genetic and environmental factors that modulate disease development. Only a few studies have addressed the functional effects of these allelic variants on ECE1 gene transcription and enzyme activity. In addition, most investigations into potential associations between disease risk and ECE1 allelic variants have been cross-sectional studies and/or limited to small series of patients; these may therefore be prone to selection bias. Furthermore, because the ECE-1 conversion target endothelin (ET-1) displays autocrine/paracrine activity, assessment of ET-1 levels in plasma may not afford an appropriate measure of ECE1 activity [16]. More investigations will be required to clarify the association between ECE-1 enzyme expression levels and disease development including LOAD.

In conclusion, we report that the ECE1 338A alleles are associated with reduced risk of LOAD development in a Chinese population.

\section{References}

[1] H.J, Amyloid, the presenilins and Alzheimer's disease. Trends Neurosci 20 (1997), 154-159.
[2] H. Funke-Kaiser, F. Reichenberger, K. Köpke, S.M. Herrmann, J. Pfeifer, H.D. Orzechowski, W. Zidek, M. Paul and E. Brand, Differential binding of transcription factor E2F-2 to the endothelin-converting enzyme-1b promoter affects blood pressure regulation, Hum Mol Genet 12 (2003), 423-433.

[3] B. Funalot, T. Ouimet, A. Claperon, C. Fallet, A. Delacourte, J. Epelbaum, T. Subkowski, N. Léonard, V. Codron, J.P. David, P. Amouyel, J.C. Schwartz and N. Helbecque, Endothelinconverting enzyme-1 is expressed in human cerebral cortex and protects against Alzheimer's disease, Mol Psychiatry 9 (2004), 1122-1128.

[4] R. Scacchi, G. Gambina, E. Broggio, M. Ruggeri and R.M. Corbo, C-338A polymorphism of the endothelin-converting enzyme (ECE-1) gene and the susceptibility to sporadic lateonset Alzheimer's disease and coronary artery disease, Disease Markers 24 (2008), 175-179.

[5] American Psychiatric Association, Diagnostic and Statistical Manual of Mental Disorders, 4th edn. American Psychiatric Association, Washington D.C. 1994.

[6] G. McKhann, D. Drachman, M. Folstein, R. Katzman, D. Price and E.M. Stadlan, Price and E.M. Stadlan, Clinical diagnosis of Alzheimer's disease: report of the NINCDS- ADRDA Work Group under the auspices of Department of Health and Human Services Task Force on Alzheimer's Disease, Neurology 34 (1984), 939-944.

[7] S.A. Miller, D.D. Dykes and H.F. Polesky, Polesky, A simple salting out procedure for extracting DNAfrom human nucleated cells, Nucleic Acids Res 16 (1988), 1215

[8] B. Funalot, D. Courbon, T. Brousseau, O. Poirier, C. Berr, F. Cambien, P. Amouyel, J.C. Schwartz and P. Ducimetière, EVA Study, Genes encoding endothelin-converting enzyme-1 and endothelin-1 interact to influence blood pressure in women: the EVA study, J Hypertens 22 (2004), 739-743.

[9] P. Garnier-Gere and C. Dillmann, A computer program for testing pairwise linkage disequilibria in subdivided populations, J Hered 83 (1992), 239.

[10] D.J. Schaid, C.M. Rowland, D.E. Tines, R.M. Jacobson and G.A. Poland, Score tests for association between traits and 
haplotypes when linkage phase is ambiguous, Am J Hum Genet 70 (2002), 425-434.

[11] K. Bühler, M. Ufer, A. Müller-Marbach, U. Brinkmann, M Laule, V. Stangl, I. Roots, K. Stangl and I. Cascorbi, Risk of coronary artery disease as influenced by variants of the human endothelin and endothelin-converting enzyme genes, Pharmacogenetics and Genomics 17 (2007), 77-83.

[12] M. Wragg, M. Hutton and C. Talbot, The Alzheimer's disease collaborative group: Genetic association between intronic polymorphism in presenilin-1 gene and late-onset Alzheimer's disease, Lancet 347 (1996), 509-512.

[13] E.A. Eckman, D.K. Reed and C.B. Eckman, Degradation of the Alzheimer's amyloid $\beta$ peptide by endothelin-converting enzyme, J Biol Chem 276 (2001), 24540-24548.

[14] N.N. Nalivaeva, L.R. Fisk, N.D. Belyaev and A.J. Turner, Amyloid-degrading enzymes as therapeutic targets in Alzheimer's disease, Curr Alzheimer Res 5(2) (Apr 2008), 212-224.

[15] L.S. Wang, N.P. Tang, H.J. Zhu, B. Zhou, L. Yang and B. Wang, Endothelin-converting enzyme-1b C-338A polymorphism is associaated with the increased risk of coronary artery disease in Chinese population, Clin Chim Acta 384 (2007), $75-79$.

[16] S.J. Pinto-Sietsma and M. Paul, A role for endothelin in the pathogenesis of 335 hypertension: fact or fiction? Kidney Int 67 (1998), S115-S121. 


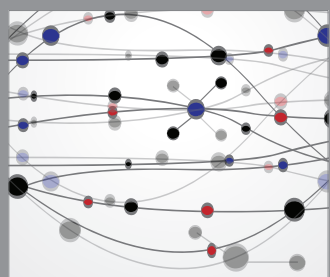

The Scientific World Journal
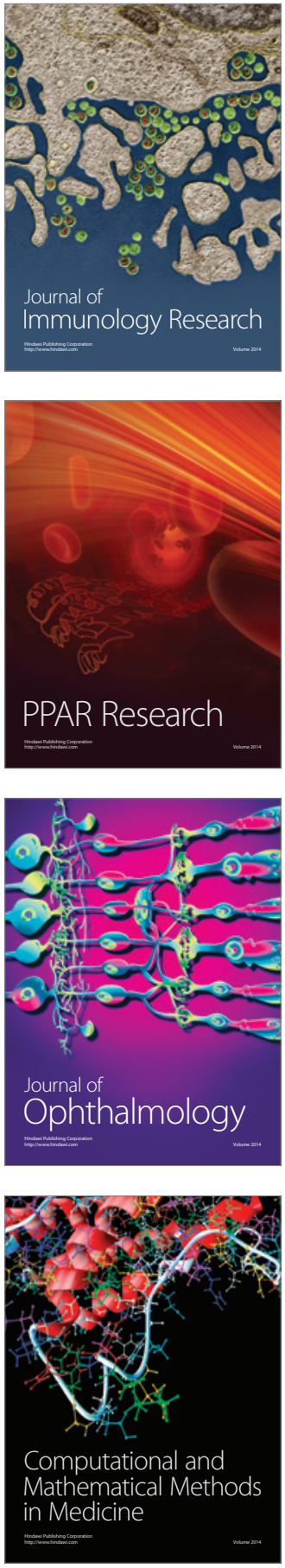

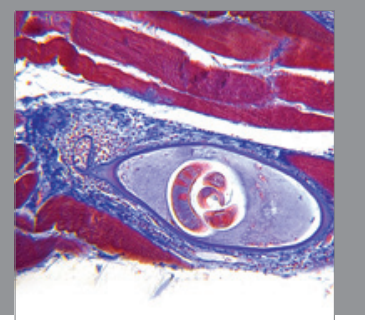

Gastroenterology

Research and Practice
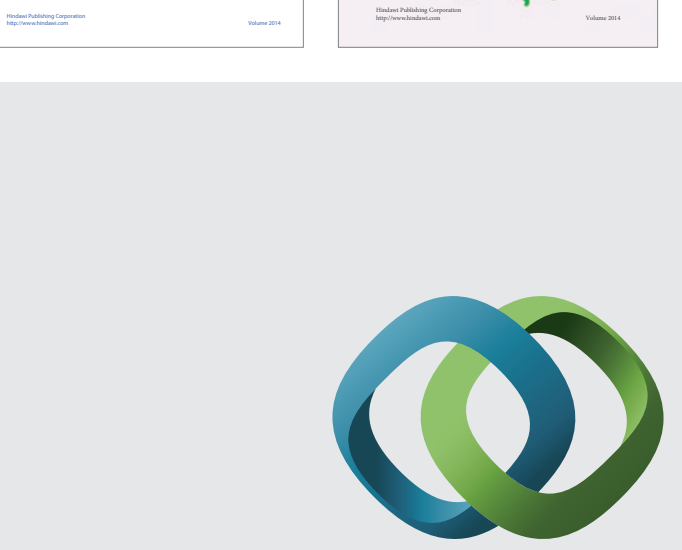

\section{Hindawi}

Submit your manuscripts at

http://www.hindawi.com
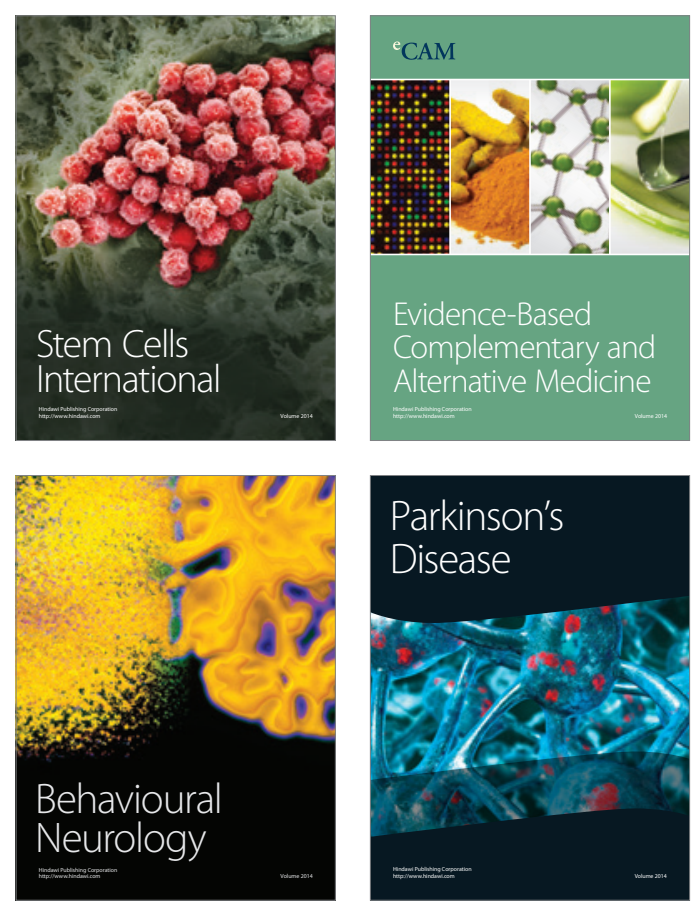

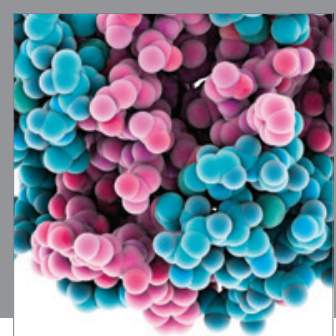

Journal of
Diabetes Research

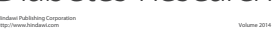

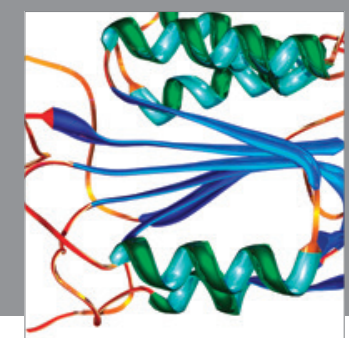

Disease Markers
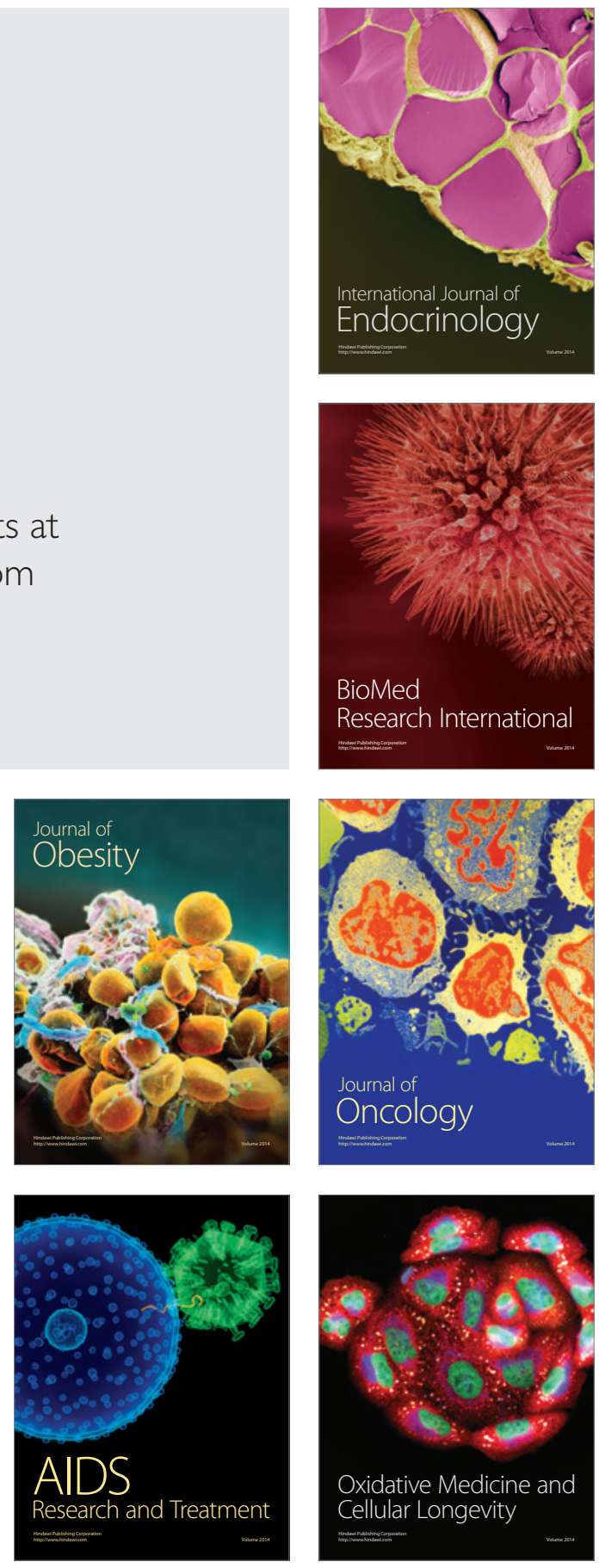